\title{
Therapeutic Role of Dance in the Treatment of Endometriosis-Related Chronic Pelvic Pain: A Proof- of-Concept Study
}

\author{
M Rius ${ }^{1}$, L Quintas ${ }^{2}$, S Kauffmann ${ }^{3}$, A Pérez ${ }^{4}$, M Ridaura ${ }^{5}$, C Gurruchaga ${ }^{6}$ and F Carmona ${ }^{1 *}$ \\ ${ }^{1}$ Benign Pathology Unit, Women's Health Institute, Spain \\ ${ }^{2}$ Pelvic Pain Unit, Women's Health Institute, Spain \\ ${ }^{3}$ Physical Therapy Unit, Women's Health Institute, Spain \\ ${ }^{4}$ Familial Therapy Unit, Psychiatry Service Hospital, Spain \\ ${ }^{5}$ Dance Studio, Doctors Trias i Pujol, Spain
}

${ }^{6}$ Asociación de Afectadas de Endometriosis de Cataluña Sardenya, Spain

*Corresponding author: F Carmona, Benign Pathology Unit Women's Health Institute Via Augusta 281, 08017, Barcelona, Spain.
Received Date: October 03, 2018

Published Date: October 12, 2018

\section{Abstract}

Background: Endometriosis causes chronic pelvic pain in many of the women affected, which may not remit after surgery or pharmacological treatment. Physical exercise as complementary therapy has already shown to be beneficial QoL in some chronic diseases, including endometriosis, but has not hitherto tested in the form of dance.

Material and Methods: A prospective cohort-proof-of-concept, open-label study, was conducted during the first half of 2017 in a private gynecologic center in Barcelona (Spain). Participants attended 12 dance sessions along eight weeks and were followed for one month after the end of the therapy. 6 nulliparous women who had been preciously treated by one or more surgeries, without current evidence of endometriosis but complaining of endometriosis-related chronic pelvic pain were included in this proof-ofconcept study. Pain, QoL and anxiety and kinesiophobia parameters were measured along the study.

Results: Results showed a benefit for the QoL parameters, being significant for the SF-36, which improved in a $20.5 \%$ for its Mental Component Summary and in a $16.4 \%$ for its Physical Component Summary. The other parameters did not reach clinical significance although improvement outcomes were observed for Zung scale and Tampa scale of kinesiophobia. Pain outcomes, measured with the Endometriosis Associated Pelvic Pain scale, did not show a significant improvement along the study.

Conclusion: Although studies with more ambitious design are needed in order to extrapolate the results of this proof of concept, physical exercise seems to have a beneficial effect on QoL of women with endometriosis-related chronic pain

Keywords: Endometriosis; Chronic pelvic pain; Physical exercise; Dancing; Quality of life

\section{Introduction}

Endometriosis is an inflammatory disease that causes chronic pelvic pain in around a great number of the women affected, which may not completely remit after applying a medical therapy or surgery in a righteous way [5]. Chronic pelvic pain is difficult to treat and one of the most prevalent symptoms related to endometriosis, together with dyspareunia; moreover, it may lead to other symptoms such as anxiety and stress that may contribute to the decrease of these patients' quality of life (QoL) [1].
Prescription of exercise as medicine and the possible mechanism of action by which it helps improving outcomes has been studied in several diseases, including psychiatric, neurological, metabolic, cardiovascular, pulmonary, musculoskeletal and cancer [2]; and great interest in new alternatives and complementary methods has increased among physicians to address the symptoms and recurrence of endometriosis. 
Dancing, a special form of movement and physical exercise, has shown to be beneficial as additional therapy in some affections such as Parkinson's disease and chronic pain [3-6]. However, no data have been published on the effect of dancing as therapy for women who suffer endometriosis-related pelvic pain.

Although the therapeutic role of physical exercise in endometriosis has been proved to improve pain outcomes, it has not been yet studied whether dance can have this beneficial effect. Dancing may contribute to diminish endometriosis-related chronic pelvic pain refractory to other treatments [7]. Secondarily, the reduction of pain may be associated to a reduction in the levels of depression and anxiety. In this proof-of-concept study, we analyses the evolution of QoL parameters in women using dancing exercises as therapy.

\section{Materials and Methods}

This open-label, single-center, non-controlled study was designed as a proof of concept to measure the change in pain and quality of life parameters before starting dance sessions and after.

\section{Selection of patients}

Six patients were included, accounting for an ideal size for a dancing therapy group. Demographic characteristics of the patients are displayed in Table 1. Patients included had previous histologic diagnosis of endometriosis and did not show any evidence of current active disease in the moment of inclusion. All of them complained of chronic pelvic pain considered as an endometriosis sequel their level of pain was severe (VAS $\geq 7$ ); they should not be attending pelvic physiotherapy or related treatments currently or three months prior to inclusion, and they had signed the informed consent. Patients were not included in the study if they experienced impossibility or difficulty of movement which makes unable to perform the provided dancing movements or if they present other medical conditions which contraindicates the performance of those movements. Before inclusion, patients were clinically evaluated at Women's Health Institute in Barcelona, with the collaboration of the Asociación de Afectadas de Endometriosis de Cataluña (Endo\&Cat).

\section{Study objectives}

All the patients were evaluated from clinical, physical, psychological and QoL's perspectives. The evaluation of endometriosis patient's status included the anamnesis, medical history, physical exploration and ultrasound study to assess the extent of the disease. Physical (ability of the patients to perform the dancing movements) and psychologic status were also assessed.

The primary endpoints were to compare the levels of pain and QoL before and after the dance sessions: Level of pain was measured using the endometriosis-associated pelvic pain (EAPP) with the use of an 11-point numeric rating scale (NRS), with 0 indicating absence of pain and 10 pain as bad as it could be for the symptoms of dysmenorrhea, dyspareunia, dyschezia, ovulatory pain and non-cyclic pelvic pain. Patients were considered eligible if they complained of at least one moderate-to-severe pain symptom (points 6-8, moderate pain; point 9 or 10, severe pain). QoL was evaluated with the 36-item short form survey (SF-36). Secondary endpoints were to assess the evolution of anxiety and stress levels using the Zung self-rating scale and the Tampa scale of kinesiophobia (TSK)-11. All the questionnaires performed where according to validated Spanish versions.

\section{Study design}

The program consisted in 12 dance sessions performed in the Dance studio in Barcelona. At weeks 1, 2, 3 and 4, women attended two sessions per week (Tuesday and Thursday); at weeks 5, 6, 7 and 8, women attended one session per weeks (Tuesday). Each session lasted 50 minutes, starting at $1 \mathrm{pm}$.

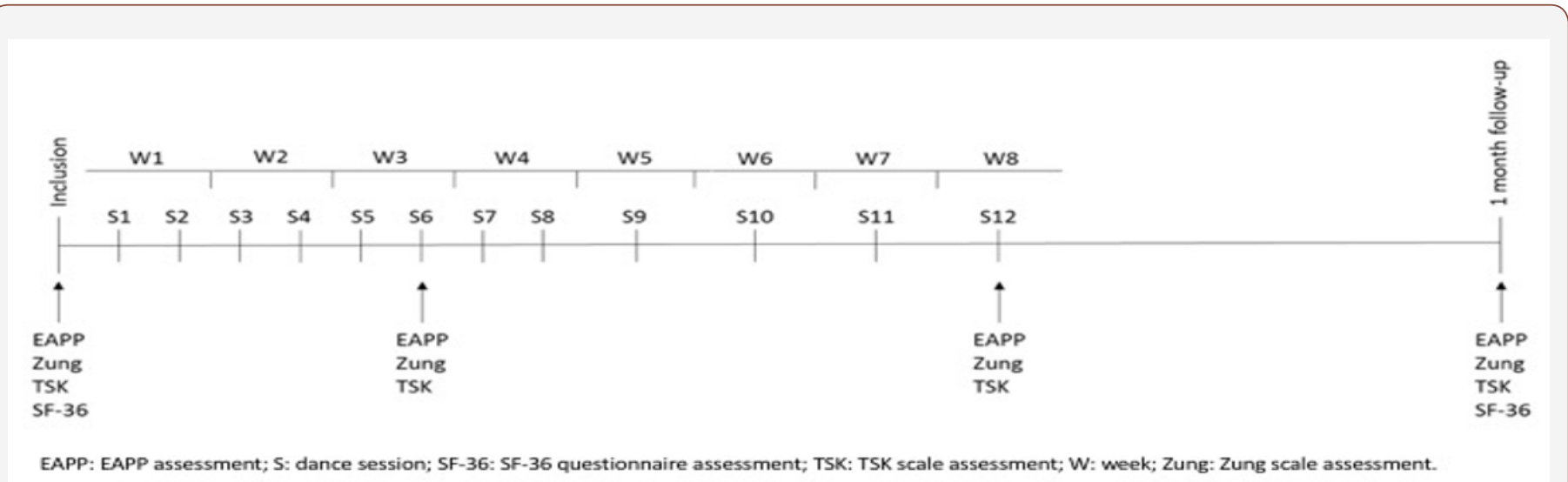

Figure 1: Study design.

Pain parameters for EAPP VAS, Zung scale and TSK-11SV were evaluated previous to inclusion, after sessions 6 and 12 and in the one-month follow-up visit. Quality of life (SF-36) and physical parameters were evaluated previous to inclusion and in the onemonth follow-up visit. A scheme of the study design is displayed in Figure 1.

\section{Sessions methodology}

Each session was scheduled in 15 minutes of warming up, 20 minutes of choreography, 10 minutes of improvisation and 5 minutes of relaxation and cooling down.

The sessions included specific movements. A description 
of performed dance movements is included in Supplementary information.

\section{Statistical analysis}

Since this study was a proof of concept, a small sample size was enough to cover the statistical objectives. This size was also suitable for a dancing group and allowed a close and thorough analysis of the outcomes. Data were collected ad-hoc and analyzed by the Fisher's exact and non-parametric tests.

\section{Results}

All participants had had at least one endometriosis-related surgery and the six of them were on hormonal treatment at the inclusion; none of them had previous births (Table 1).

Table 1: Demographic characteristics of the patients at the moment of inclusion.

\begin{tabular}{|c|c|}
\hline Variable & \\
\hline Age, years Mean (range) & $37.2(35-42)$ \\
\hline Parity, nr of births Mean (range) & 0 \\
\hline $\begin{array}{c}\text { Time from first symptom to diagnostic, years- } \\
\text { Mean (range) Number of previous surgeries* }\end{array}$ & $10.2(1-20)$ \\
\hline Total mean (range) & $3.1(1-5)$ \\
\hline Exploratory laparoscopy & 2 \\
\hline Endometrioma excision & 4 \\
\hline Unilateral adnexectomy & 1 \\
\hline Deep nodule excision & 6 \\
\hline Adenomyoma excision & 1 \\
\hline Hysterectomy + bilateral adnexectomy & 1 \\
\hline Number of current endometriosis treatment & 2 \\
\hline Oral progestin & 1 \\
\hline Mirena® (intrauterine device) & 3 \\
\hline Combined oral contraceptives & \\
\hline
\end{tabular}

${ }^{*}$ A patient may have more than one previous surgical procedure Table 2: Evolution of analyzed parameters

\begin{tabular}{|c|c|c|c|c|c|}
\hline Parameter & $\begin{array}{l}\text { Base- } \\
\text { line }\end{array}$ & Session 6 & $\begin{array}{c}\text { Session } \\
12\end{array}$ & $\begin{array}{c}\text { 1-month } \\
\text { fol- } \\
\text { low-up }\end{array}$ & $\begin{array}{c}\mathbf{p} \\
\text { value }\end{array}$ \\
\hline $\begin{array}{l}\text { Maximum } \\
\text { EAPP }\end{array}$ & $\begin{array}{l}8.7(7- \\
10)\end{array}$ & $8.2(7-10)$ & $\begin{array}{l}9.3(7- \\
10)\end{array}$ & $\begin{array}{c}9.0(7- \\
10)\end{array}$ & 0.8 \\
\hline \multicolumn{6}{|l|}{ Zung scale } \\
\hline Depression & $\begin{array}{c}57.5 \\
(43-68)\end{array}$ & $\begin{array}{l}55.4(41- \\
64)\end{array}$ & $\begin{array}{c}52.3 \\
(40-62)\end{array}$ & $\begin{array}{c}51.9(39- \\
61)\end{array}$ & 0.3 \\
\hline Anxiety & $\begin{array}{c}61.3 \\
(42-69)\end{array}$ & $\begin{array}{c}58.4(44- \\
65)\end{array}$ & $\begin{array}{c}55.5 \\
(40-63)\end{array}$ & $\begin{array}{c}56.4(39- \\
65)\end{array}$ & 0.2 \\
\hline TSK & $\begin{array}{c}25.2 \\
(21-36)\end{array}$ & $\begin{array}{c}24.6(21- \\
32)\end{array}$ & $\begin{array}{c}23.1 \\
(19-28)\end{array}$ & $\begin{array}{l}20.7 \text { (16- } \\
29)\end{array}$ & 0.06 \\
\hline \multicolumn{6}{|l|}{ SF-36 } \\
\hline MCS & $\begin{array}{c}64.1 \\
(49-84)\end{array}$ & - & - & $\begin{array}{c}80.6(59- \\
87)\end{array}$ & $<0.05$ \\
\hline PCS & $\begin{array}{c}68.9 \\
(53-81)\end{array}$ & - & - & $\begin{array}{c}82.4(59- \\
89)\end{array}$ & $<0.05$ \\
\hline
\end{tabular}

No women discontinued the therapy. $\mathrm{N}=6$ for all the timepoints assessed.

MCS: mental component summary; PCS: physical component summary. Values are mean (range).
Table 2 summarizes the more important study findings. Zung anxiety and pain parameters did not show a clear tendency towards improvement during the study period. However, a clear (although not statistically significant) trend to improvement in patient's mobility was observed with a $20 \%$ decrease in the TSK- 11 scale.

Improvements for all the measured QoL parameters were observed from baseline to the end of the study Statistical significance was reached for improvements in the SF-36 global QoL scores measured at baseline and at the end of the study for both its mental (MCS) and physical (PCS) components, with a slightly better performance for the MCS: 16.5 points (20.5\%) vs. 13.5 points (16.4\%) for PCS.

\section{Discussion}

This is the first study analyzing the effect of dancing in endometriosis-related chronic pelvic pain. Significant improvements in SF-36 scale seem to indicate a better performance in QoL after the dance therapy. Improvement in parameters such as TSK and Zung, although non-significant, sum to the fact that dance as complementary therapy may represent a good tool to address endometriosis-related complications. Although the reported endometriosis-related pain scores did not improve during the study, it is worth to notice that the main objective of this proof of concept was to assess QoL, which improved for all the parameters measured: SF-36, Zung and TSK.

Several studies have been published recently analyzing the role of physical therapies and moderate exercise in improving pain and QoL outcomes of women affected. Carpenter, et al. [8] reported a reduction in the number of androgenic side effects in women with endometriosis treated with danazol and physical exercise compared with women receiving only danazol. More recently, Zhao L et al. [9] probed an improvement of anxiety, depression and QoL outcomes in Chinese women with endometriosis under gonadotropin-releasing hormone agonist therapy who practiced progressive muscular relaxation training for 12 weeks. Similar data were obtained by Friggi K, et al. [4] [10] A review from Bonocher $\mathrm{CM}$, et al. [11] claimed that regular exercise has protective effect against inflammatory diseases and reduces estrogen levels. Yoga has been also used with positive results in Brazilian patients as a form of exercise integrating body and mind [1,12].

The results of this study confirm those obtained by Gonçalves mind [1,12], showing the beneficial effect of physical exercise in QoL and pain parameters among women who suffer endometriosis. They also add a first glimpse of the role of dancing as complementary therapy for the treatment in this disease.

Endometriosis-related complications still represent a challenge in clinical practice mind [1]. Severe pain is associated with endometriosis at a high rate and caused by an interaction of factors such as mechanical interactions, inflammation, immune system and nerve fibers [1]. The connection of these nerve fibers to the central nervous system (CNS) has been shown to create a permanent stimulus which may persist even after the removal of the endometrial lesion [13]. Since pharmacological treatment addressing the physical pathways of pain seem not to control 
efficiently enough this complication, several researches try to improve women's QoL by addressing the psychological side of pain [1].

Since severe pain seems to have a complex building into the CNS [13], the slower change in EAPP scores and the slightly better improvement in the MCS- over the PCS-SF-36 scale may be explained by its characteristic persistency. A long-term design is needed in order to analyses the influence that improving of QoL has in reversing the neurological stimuli leading to chronic pain. It is worth to notice that last assessments were performed one month after finishing the therapy and the improvements were registered even though no more dance sessions were performed (physical activity of each women outside of the study was not recorded).

Endometriotic pelvic pain has been also associated with adenomyosis [14]. Being the myometrium involved, it can be hypothesized that movements such as the employed in dance sessions, which help to gain muscular elasticity and strength around the uterine area, may provide a benefit in women's pain and QoL outcomes.

One of the most noticeable limitations of this study was its size, since the number of patients recruited depended on the optimal sample size of a dance session. Due to the novelty of a study with these characteristics, an optimal sample size cannot be determined based on previous studies, and it was decided based on the most beneficial number for performance. The lack of control group and the open character may compromise the strength of the results. Besides, movements performed by each patient may be biased by their own capacities, mood and physical abilities. On the other hand a small number of patients allowed for proximity and enough time for exploration and measurements, which benefits the outcomes accuracy.

Since few patients were recruited, the study is only exploratory, and our results need to be confirmed in larger and more heterogeneous population studies. More studies on dance and endometriosis are required to support our results and give a clearer idea of the benefits dance can offer as complementary therapy for women who suffer endometriosis-related pelvic pain despite righteous surgical and/or pharmacological treatment.

\section{Acknowledgement}

The authors thank staff members from our institutions for their assistance with data collection and patients care. The authors also gratefully acknowledge Dr. Izaskun Muruzábal-Lecumberri for her collaboration in writing this paper.

\section{References}

1. Gonçalves AV, Barros NF, Bahamondes L (2017) The Practice of hatha yoga for the treatment of pain associated with endometriosis. J Altern Complement Med 23(1): 45-52.

2. Pedersen BK, Saltin B (2015) Exercise as medicine - evidence for prescribing exercise as therapy in 26 different chronic diseases. Scand J Med Sci Sports 25 Suppl 3: 1-72.

3. Butt CA (2017) "Move your arm like a swan": dance for pd demedicalizes parkinson disease. JAMA 317(4): 342-343.

4. Hulbert S, Ashburn A, Roberts L, Verheyden G (2017) Dance for parkinson's-the effects on whole body co-ordination during turning around. Complement Ther Med 32: 91-97.

5. Shanahan J, Morris ME, Bhriain ON, Volpe D, Lynch T, et al. (2017) Dancing for parkinson disease: a randomized trial of irish set dancing compared with Usual Care. Arch Phys Med Rehabil 98(9): 1744-1751.

6. Ventura MI, Barnes DE, Ross JM, Lanni KE, Sigvardt KA, et al. (2016) A pilot study to evaluate multi-dimensional effects of dance for people with Parkinson's disease. Contemp Clin Trials 51: 50-55.

7. Krampe J, Wagner JM, Hawthorne K, Sanazaro D, Wong-Anuchit C, et al. (2014) Does dance-based therapy increase gait speed in older adults with chronic lower extremity pain: a feasibility study. Geriatr Nurs 35(5): 339-344

8. Carpenter SE, Tjaden B, Rock JA, Kimball A (1995) The effect of regular exercise on women receiving danazol for treatment of endometriosis. Int J Gynaecol Obstet 49(3): 299-304.

9. Zhao L, Wu H, Zhou X, Wang Q, Zhu W, et al. (2012) Effects of progressive muscular relaxation training on anxiety, depression and quality of life of endometriosis patients under gonadotrophin-releasing hormone agonist therapy. Eur J Obstet Gynecol Reprod Biol 162(2): 211-215.

10. Friggi K, Garcia MC, Petta CA, Ribeiro DA, de Oliveira Monteiro NR, et al. (2012) Physical therapy and psychological intervention normalize cortisol levels and improve vitality in women with endometriosis. J Psychosom Obstet Gynaecol 33(4): 191-198.

11. Bonocher CM, Montenegro ML, Rosa E Silva JC, Ferriani RA, Meola J (2014) Endometriosis and physical exercises: a systematic review. Reprod Biol Endocrinol 12: 4.

12. Gonçalves AV, Makuch MY, Setubal MS, Barros NF, Bahamondes L (2016) A Qualitative study on the practice of yoga for women with painassociated endometriosis. J Altern Complement Med 22(12): 977-982.

13. Stratton P, Khachikyan I, Sinaii N, Ortiz R, Shah J (2015) Association of chronic pelvic pain and endometriosis with signs of sensitization and myofascial pain. Obstet Gynecol 125(3): 719-728.

14. Perelló MF, Martínez-Zamora MÁ, Torres X, Munrós J, Balasch Cortina J (2017) Endometriotic pain Is associated with adenomyosis but not with the compartments affected by deep Infiltrating endometriosis. Gynecol Obstet Invest 82(3): 240-246. 